\title{
Analytical Models of Exoplanetary Atmospheres. V. Non-gray Thermal Structure with Coherent Scattering
}

\author{
Gopakumar Mohandas $^{1,2}$ (1D), Martin E. Pessah ${ }^{1}$ (D) and Kevin Heng ${ }^{3}$ (i) \\ ${ }^{1}$ Niels Bohr International Academy, Niels Bohr Institute, Blegdamsvej 17, DK-2100 Copenhagen Ø, Denmark; gopakumar@nbi.ku.dk \\ ${ }^{2}$ Kavli Institute for Theoretical Physics, University of California, Santa Barbara 93106, USA \\ ${ }^{3}$ University of Bern, Center for Space and Habitability, Gesellschaftsstrasse 6, CH-3012, Bern, Switzerland \\ Received 2018 February 2; accepted 2018 February 26; published 2018 April 26
}

\begin{abstract}
We apply the picket fence treatment to model the effects brought about by spectral lines on the thermal structure of irradiated atmospheres. The lines may be due to pure absorption processes, pure coherent scattering processes, or some combination of absorption and scattering. If the lines arise as a pure absorption process, the surface layers of the atmosphere are cooler, whereas this surface cooling is completely absent if the lines are due to pure coherent isotropic scattering. The lines also lead to a warming of the deeper atmosphere. The warming of the deeper layers is, however, independent of the nature of line formation. Accounting for coherent isotropic scattering in the shortwave and longwave continuum results in anti-greenhouse cooling and greenhouse warming on an atmosphere-wide scale. The effects of coherent isotropic scattering in the line and continuum operate in tandem to determine the resulting thermal structure of the irradiated atmosphere.
\end{abstract}

Key words: methods: analytical - planets and satellites: atmospheres - radiative transfer

\section{Introduction}

Analytical radiative transfer models have proved to be of considerable utility in the study of stellar and planetary atmospheres since their development a century ago (Mihalas 1970). Despite the availability of sophisticated high-speed numerical techniques in the present day (Hubeny 2017), simplified analytical treatments continue to remain valuable primarily as a means to derive physical insight and understanding of atmospheric conditions.

The usefulness of analytical models to construct model atmospheres are afforded by the simplistic nature of the underlying assumptions, namely that of gray opacities, planeparallel steady-state structure, hydrostatic and radiative equilibrium (Mihalas 1970). These assumptions are, however, also the source of their limitations. In particular, the assumption of frequency independent (gray) mean opacities are far from a realistic representation of true atmospheric opacities (Heng 2017). Nevertheless, such simplified 1D analytical models have been useful in providing an exact solution that predicts basic atmospheric trends and one that may serve as a good initial approximation for numerical schemes (Hubeny \& Mihalas 2014).

Analytical or semi-analytical models with small departures from grayness have been derived over the years (Hubeny \& Mihalas 2014). In the context of irradiated atmospheres, such as that of close-in extrasolar planets, the most elementary extension constitutes what is referred to as the two-step gray or semi-gray or dual-band transfer models (Hansen 2008; Guillot 2010; Heng et al. 2012, 2014). These models are predicated on the approximate division of radiant energy into two distinct and nearly nonoverlapping bands; the shortwave associated with external stellar irradiation and the longwave associated with internal planetary thermal emission. The transfer equations or their moments, with different mean opacities for the shortwave and longwave components, are then solved separately and linked together by the principle of energy conservation.
Recently, Parmentier \& Guillot (2014) derived an analytical model by applying the picket fence method of Chandrasekhar (1935) to irradiated atmospheres. The picket fence treatment was originally developed to model line blanketing effects in nonirradiated stellar atmospheres and has since been refined by a number of authors (Münch 1946; Athay \& Skumanich 1969; Mihalas \& Luebke 1971). Spectral line blanketing leads to two major effects that introduces departures from a gray atmosphere: surface cooling and backwarming (Mihalas \& Luebke 1971; Athay 2012). The former refers to the reduction of temperature in the upper layers of the atmosphere due to the added emissivity of the lines whereas the latter effect describes the temperature enhancement in the deeper atmosphere as a result of the flux redistribution within the continuum due to the lines. Both of these effects were present in the model derived by Parmentier \& Guillot (2014). However, their analysis did not account for the influence of scattering in both the shortwave and the longwave. The surface cooling effect has been seen to be dependent on the nature of line formation in nonirradiated atmospheres (Chandrasekhar 1935). The degree of cooling is lower if scattering contributes to the line in some measure and is completely absent when the lines are entirely due to a pure scattering process. One would expect this feature to be present in irradiated atmospheres as well. Furthermore, continuum scattering is known to induce an atmospherewide shift in temperatures (Heng et al. 2014; Heng 2017). This shift is toward hotter temperatures if the isotropic scattering contributes to the longwave continuum and is toward lower temperatures if isotropic scattering contributes to the shortwave continuum. Therefore, one must account for scattering in the line and continuum in order to derive a closer approximation to actual atmospheric thermal structures.

In this paper, we generalize the picket fence treatment to irradiated atmospheres to include coherent scattering effects. We derive solutions that accommodate for coherent isotropic scattering in the lines as well as the continuum, in the longwave and shortwave frequency bands. Our model therefore provides a fuller picture of the possible atmospheric thermal structure 
while preserving the advantages and utility rendered by tractable nongray analytical models.

The paper is organized as follows. In Section 2, we present the picket fence model equations. In Section 3, we connect the longwave picket fence model with the shortwave equations and solve for the resulting temperature profile. We present a discussion of the results in Section 4 and conclude with a summary in Section 5.

\section{The Picket Fence Model}

We consider a plane-parallel atmosphere and model the transport of radiation by solving the moments of the steadystate radiative transfer equation. We apply the dual-band approximation and derive separate moment equations in the longwave and shortwave frequency bands.

We begin with the longwave band where we make use of the picket fence method (Chandrasekhar 1935). The radiative transfer equation in a plane-parallel atmosphere has the basic form

$$
\mu \frac{\partial I_{\nu}}{\partial m}=k(\nu)\left(I_{\nu}-S_{\nu}\right),
$$

where $\mu$ is the cosine of the zenith angle, $m$ is the column mass, $k$ is the frequency dependent extinction opacity, $I_{\nu}$ is the specific intensity, and $S_{\nu}$ is the source function. The intensity and source function are, in general, functions of $\mu, m$, and $\nu$.

We define the moments of the intensity, the mean intensity, the Eddington flux, and the $K$-integral, respectively, as follows (Mihalas 1970)

$$
\begin{aligned}
J_{\nu} & \equiv \frac{1}{2} \int_{-1}^{1} I_{\nu}(\tau, \mu) d \mu, \\
H_{\nu} & \equiv \frac{1}{2} \int_{-1}^{1} I_{\nu}(\tau, \mu) \mu d \mu, \\
K_{\nu} & \equiv \frac{1}{2} \int_{-1}^{1} I_{\nu}(\tau, \mu) \mu^{2} d \mu .
\end{aligned}
$$

In the parts of the frequency interval containing lines, the equation of transfer is (Athay 2012; Mihalas 1970)

$$
\mu \frac{\partial I_{\nu}}{\partial m}=\left(k_{c}+k_{l}\right) I_{\nu}-\left(k_{c} S_{c \nu}+k_{l} S_{l \nu}\right),
$$

where the subscripts $c$ and $l$ refer to the continuum and line respectively. Considering coherent isotropic scattering, the continuum and line source functions have the form

$$
\begin{array}{cc}
S_{c \nu}=\varepsilon_{c} B_{\nu}+\left(1-\varepsilon_{c}\right) J_{\nu}, & \varepsilon_{c}=\frac{\kappa_{c}}{k_{c}}, \\
S_{l \nu}=\varepsilon_{l} B_{\nu}+\left(1-\varepsilon_{l}\right) J_{\nu}, & \varepsilon_{l}=\frac{\kappa_{l}}{k_{l}},
\end{array}
$$

where $\kappa$ denotes the absorption opacity that together with the corresponding scattering opacity assume constant but separate values in the line and continuum. The parameter $\varepsilon$ is a measure of the fraction of photons lost to pure absorption (Mihalas 1970). It is in fact the complementary parameter to the single scattering albedo but we shall refer to it here as the scattering parameter regardless.
Integrating over the frequency interval containing only the lines leads to the transfer equation

$$
\begin{aligned}
\mu \frac{\partial I_{1}}{\partial \tau}= & \left(\frac{1}{\varepsilon_{c}}+\xi\right) I_{1}-\left[\frac{1}{\varepsilon_{c}}-1+\left(1-\varepsilon_{l}\right) \xi\right] J_{1} \\
& -\left(1+\varepsilon_{l} \xi\right) \beta \mathcal{B},
\end{aligned}
$$

whereas integrating over the remainder of the frequency interval representing the continuum yields

$$
\varepsilon_{c} \mu \frac{\partial I_{2}}{\partial \tau}=I_{2}-\left(1-\varepsilon_{c}\right) J_{2}-(1-\beta) \varepsilon_{c} \mathcal{B} .
$$

Here the subscripts 1 and 2 represent the integrated variables in the line and continuum respectively. We have also defined the line to continuum opacity ratio

$$
\xi=\frac{k_{l}}{\kappa_{c}},
$$

the integrated blackbody function

$$
\mathcal{B}=\int B_{\nu} d \nu,
$$

and the frequency independent optical depth

$$
d \tau=\kappa_{c} d m
$$

Finally, we introduce the parameter $\beta$, which gives the relative probability of finding a line in the frequency interval and may in general be a function of frequency. However, we take $\beta$ to be a constant in Equations (8) and (9) assuming that the lines have uniform width and are uniformly spread across the spectral range.

The first and second moments of the radiative transfer equation in the line and continuum are, respectively,

$$
\begin{gathered}
\frac{d H_{1}}{d \tau}=\lambda\left(J_{1}-\beta \mathcal{B}\right), \\
\frac{d H_{2}}{d \tau}=J_{2}-(1-\beta) \mathcal{B},
\end{gathered}
$$

and

$$
\begin{aligned}
\frac{d K_{1}}{d \tau} & =\frac{\eta}{\varepsilon_{c}} H_{1}, \\
\frac{d K_{2}}{d \tau} & =\frac{1}{\varepsilon_{c}} H_{2},
\end{aligned}
$$

where we have defined

$$
\lambda=1+\varepsilon_{l} \xi, \quad \eta=1+\varepsilon_{c} \xi .
$$

In the limit $\varepsilon_{c}=1$, we recover the moment equations used by Chandrasekhar (1935).

\section{Irradiated Atmospheres}

We now extend the original picket fence treatment to irradiated atmospheres, like that of close-in extrasolar giant planets. This is achieved by linking the shortwave transfer solution to the longwave picket fence model solution via the radiative equilibrium condition.

We first consider the radiative transfer equation as it applies to the shortwave frequencies. The frequency integrated transfer equation for the shortwave band, where the source function contains only a non-negligible contribution due to coherent 
isotropic scattering, is given by

$$
\mu \frac{d I_{s}}{d \tau}=\frac{\gamma}{\varepsilon_{s}}\left[I_{s}-\left(1-\varepsilon_{s}\right) J_{s}\right],
$$

with

$$
\gamma=\frac{\kappa_{s}}{\kappa_{c}}, \quad \varepsilon_{s}=\frac{\kappa_{s}}{k_{s}},
$$

where $\kappa_{s}$ and $k_{s}$ are the shortwave absorption and extinction opacities respectively. The parameter $\gamma$ quantifies the strength of the shortwave opacity to its longwave continuum counterpart and $\varepsilon_{s}$ measures the fraction of shortwave photons lost to absorption. The moment equations for the shortwave are

$$
\frac{d H_{s}}{d \tau}=\gamma J_{s}
$$

and

$$
\frac{d K_{s}}{d \tau}=\frac{\gamma}{\varepsilon_{s}} H_{s}
$$

Using the closure relation $K_{s}=\bar{\mu}^{2} J_{s}$ (Guillot 2010; Heng et al. 2012, 2014), where $\bar{\mu}$ is the cosine of the angle of the collimated stellar beam with respect to the vertical, we obtain the second-order ordinary differential equation

$$
\frac{d^{2} J_{s}}{d \tau^{2}}=\frac{\gamma_{\mu}^{2}}{\varepsilon_{s}} J_{s},
$$

which has the simple exponential solution

$$
J_{s}=J_{s}(0) \exp \left(-\gamma_{\mu \varepsilon} \tau\right),
$$

where $\gamma_{\mu}=\gamma /|\bar{\mu}|$ and $\gamma_{\mu \varepsilon}=\gamma_{\mu} / \sqrt{\varepsilon_{s}}$ are assumed constant. Consistency with Equation (20) implies

$$
H_{s}=H_{s}(0) \exp \left(-\gamma_{\mu \varepsilon} \tau\right)
$$

with $H_{s}(0)=-|\bar{\mu}| \sqrt{\varepsilon_{s}} J_{s}(0)$ Heng et al. (2014).

The radiative equilibrium condition, which is given by $d\left(H_{1}+H_{2}+H_{s}\right) / d \tau=0$ implies

$$
\lambda J_{1}+J_{2}+\gamma J_{s}=[\lambda \beta+1-\beta] \mathcal{B},
$$

Adding Equations (13) and (14), we have

$$
\frac{d}{d \tau}\left(H_{1}+H_{2}\right)=-\gamma J_{s}=\gamma_{\mu \varepsilon} H_{s},
$$

which has the full solution

$$
H_{1}+H_{2}=\mathcal{H}-H_{s} .
$$

where $\mathcal{H}$ is the total integrated longwave Eddington flux. Combining Equations (15) and (16), we obtain

$$
\frac{d}{d \tau}\left(\frac{K_{1}}{\eta}+K_{2}\right)=\frac{\mathcal{H}}{\varepsilon_{c}}-\frac{1}{\varepsilon_{c}} H_{s},
$$

which has the full solution

$$
\frac{K_{1}}{\eta}+K_{2}=\frac{\mathcal{H}}{\varepsilon_{c}} \tau+c+\frac{1}{\varepsilon_{c} \gamma_{\mu \varepsilon}} H_{s} .
$$

With the Eddington approximation $J_{1,2}=3 K_{1,2}$, we may express the integrated Planck function as

$$
\mathcal{B}=\frac{3}{\tilde{\lambda}}\left[\lambda K_{1}+K_{2}-\frac{\gamma_{\mu \varepsilon}}{3} H_{s}\right] .
$$

Using Equation (29), this may be written in either of the two forms given below

$$
\begin{aligned}
& \mathcal{B}=\frac{3}{\tilde{\lambda}}\left[\frac{\mathcal{H}}{\varepsilon_{c}} \tau+c+\left(\lambda-\frac{1}{\eta}\right) K_{1}+\left(\frac{1}{\varepsilon_{c} \gamma_{\mu \varepsilon}}-\frac{\gamma_{\mu \varepsilon}}{3}\right) H_{s}\right], \\
& \mathcal{B}=\frac{3}{\tilde{\lambda}}\left[\lambda \eta\left(\frac{\mathcal{H}}{\varepsilon_{c}} \tau+c\right)+(1-\lambda \eta) K_{2}+\left(\frac{\lambda \eta}{\varepsilon_{c} \gamma_{\mu \varepsilon}}-\frac{\gamma_{\mu \varepsilon}}{3}\right) H_{s}\right] .
\end{aligned}
$$

Combining Equation (15) with Equation (13) and Equation (16) with Equation (14) by using the Eddington approximation, we obtain the pair of inhomogeneous secondorder ordinary differential equations

$$
\begin{gathered}
\frac{\varepsilon_{c} d^{2} K_{1}}{d \tau^{2}}=\eta \lambda\left(3 K_{1}-\beta \mathcal{B}\right), \\
\frac{\varepsilon_{c} d^{2} K_{2}}{d \tau^{2}}=3 K_{2}-(1-\beta) \mathcal{B} .
\end{gathered}
$$

Substituting Equations (31) and (32) in Equations (33) and (34) yields

$$
\begin{gathered}
\frac{d^{2} K_{1}}{d \tau^{2}}=\frac{3 \lambda \tilde{\eta}}{\varepsilon_{c} \tilde{\lambda}} K_{1}-\frac{3 \eta \lambda \beta}{\varepsilon_{c}^{2} \tilde{\lambda}}(\mathcal{H} \tau+c) \\
-\frac{3 \eta \lambda \beta}{\varepsilon_{c} \tilde{\lambda}}\left(\frac{1}{\varepsilon_{c} \gamma_{\mu \varepsilon}}-\frac{\gamma_{\mu \varepsilon}}{3}\right) H_{s}, \\
\frac{d^{2} K_{2}}{d \tau^{2}}=\frac{3 \lambda \tilde{\eta}}{\varepsilon_{c} \tilde{\lambda}} K_{2}-\frac{3 \eta \lambda(1-\beta)}{\varepsilon_{c}^{2} \tilde{\lambda}}(\mathcal{H} \tau+c) \\
-\frac{3(1-\beta)}{\varepsilon_{c} \tilde{\lambda}}\left(\frac{\lambda \eta}{\varepsilon_{c} \gamma_{\mu \varepsilon}}-\frac{\gamma_{\mu \varepsilon}}{3}\right) H_{s},
\end{gathered}
$$

where we have defined the convenient shorthands

$$
\begin{gathered}
\tilde{\lambda}=\lambda \beta+1-\beta, \\
\tilde{\eta}=\beta+\eta(1-\beta) .
\end{gathered}
$$

Bounded solutions to Equations (35) and (36) are given by

$$
\begin{aligned}
K_{1}= & a \exp (-q \tau)+\frac{\eta \beta}{\varepsilon_{c} \tilde{\eta}}(\mathcal{H} \tau+c) \\
& -\frac{3 \lambda \eta \beta}{\gamma_{\mu \varepsilon}^{2} \varepsilon_{c} \tilde{\lambda}-3 \lambda \tilde{\eta}}\left(\frac{1}{\varepsilon_{c} \gamma_{\mu \varepsilon}}-\frac{\gamma_{\mu \varepsilon}}{3}\right) H_{s}, \\
K_{2}= & b \exp (-q \tau)+\frac{\eta(1-\beta)}{\varepsilon_{c} \tilde{\eta}}(\mathcal{H} \tau+c) \\
& -\frac{3(1-\beta)}{\gamma_{\mu \varepsilon}^{2} \varepsilon_{c} \tilde{\lambda}-3 \lambda \tilde{\eta}}\left(\frac{\lambda \eta}{\varepsilon_{c} \gamma_{\mu \varepsilon}}-\frac{\gamma_{\mu \varepsilon}}{3}\right) H_{s},
\end{aligned}
$$

where $q$, its inverse rather, is a characteristic optical depth that is given by

$$
q=\sqrt{\frac{3 \lambda \tilde{\eta}}{\varepsilon_{c} \tilde{\lambda}}}
$$

In order for Equations (39) and (40) to add up to Equation (29), we require $a=-b \eta$. Finally, the integrated Planck function 


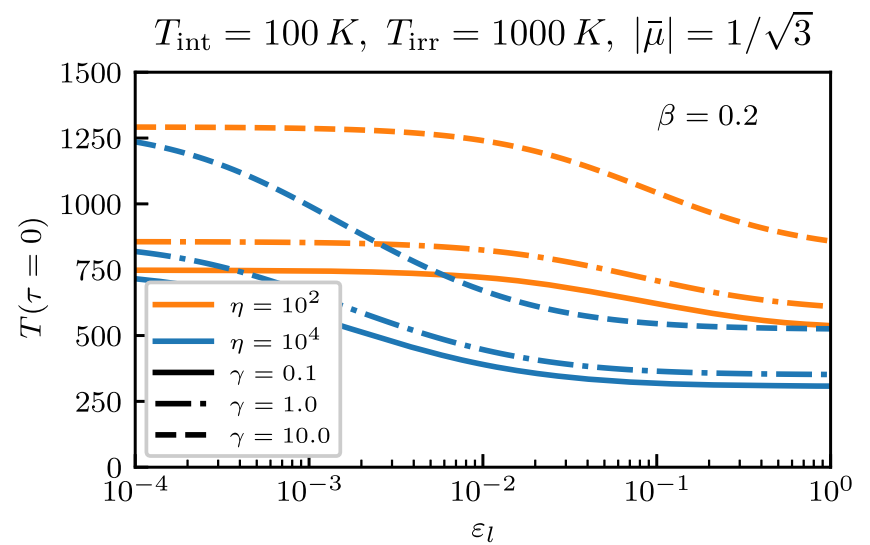

Figure 1. Boundary temperature $T(\tau=0)$ of an irradiated atmosphere as a function of the line scattering parameter $\varepsilon_{l}$ at a fixed line width $\beta$ but different values of the line strength $\eta$ and shortwave absorption opacity parameter $\gamma$.

may be expressed as

$$
\begin{aligned}
\mathcal{B}= & \frac{3 \eta}{\varepsilon_{c} \tilde{\eta}}(\mathcal{H} \tau+c)-\frac{3(\lambda \eta-1)}{\tilde{\lambda}} b \exp (-q \tau) \\
& -\frac{\left(\gamma_{\mu \varepsilon}^{2} \varepsilon_{c}-3\right)\left(\gamma_{\mu \varepsilon}^{2} \varepsilon_{c}-3 \eta \lambda\right)}{\gamma_{\mu \varepsilon} \varepsilon_{c}\left(\gamma_{\mu \varepsilon}^{2} \varepsilon_{c} \tilde{\lambda}-3 \lambda \tilde{\eta}\right)} H_{s}(0) \exp \left(-\gamma_{\mu \varepsilon} \tau\right)
\end{aligned}
$$

Thus it remains only to determine the integration constants $b$ and $c$, which we achieve by the application of suitable boundary conditions. For the sake of conformity with Chandrasekhar (1935), we use the relations $\mathcal{F}=4 \mathcal{H}$ and $\mathcal{F}_{*}=4 \mathrm{H}_{s}(0)$. Assuming that at $\tau=0$, we have $4 H_{1,2}(0)=$ $6 K_{1,2}(0)$ (Chandrasekhar 1935) and by considering Equations (15) and (16) evaluated at $\tau=0$, we obtain

$$
\begin{aligned}
& (6 \eta+4 q) b-\frac{6 \eta \beta}{\varepsilon_{c} \tilde{\eta}} c+\frac{\beta}{\varepsilon_{c} \tilde{\eta}} \mathcal{F} \\
& +\frac{3 \lambda \beta\left(\gamma_{\mu \varepsilon}+\frac{3}{2} \eta\right)}{\gamma_{\mu \varepsilon}^{2} \varepsilon_{c} \tilde{\lambda}-3 \lambda \tilde{\eta}}\left(\frac{1}{\varepsilon_{c} \gamma_{\mu \varepsilon}}-\frac{\gamma_{\mu \varepsilon}}{3}\right) \mathcal{F}_{*}=0, \\
& (6+4 q) b+\frac{6 \eta(1-\beta)}{\varepsilon_{c} \tilde{\eta}} c-\frac{\eta(1-\beta)}{\varepsilon_{c} \tilde{\eta}} \mathcal{F} \\
& -\frac{3(1-\beta)\left(\gamma_{\mu \varepsilon}+\frac{3}{2}\right)}{\gamma_{\mu \varepsilon}^{2} \varepsilon_{c} \tilde{\lambda}-3 \lambda \tilde{\eta}}\left(\frac{\lambda \eta}{\varepsilon_{c} \gamma_{\mu \varepsilon}}-\frac{\gamma_{\mu \varepsilon}}{3}\right) \mathcal{F}_{*}=0 .
\end{aligned}
$$

The boundary condition used here corresponds to the application of the second and third choices listed in Parmentier \& Guillot (2014, see their Section 2.3.2) with the second Eddington coefficient $f_{\mathrm{H}}=1 / 2$. Solving the system of Equations (43) and (44) results in

$$
b=b_{l} \mathcal{F}+b_{s} \mathcal{F}_{*}, \quad c=c_{l} \mathcal{F}+c_{s} \mathcal{F}_{*},
$$

where

$$
\begin{gathered}
b_{l}=\frac{\beta(1-\beta)(\eta-1)}{\varepsilon_{c} \tilde{\eta}(6 \tilde{\eta}+4 q)}, \\
b_{s}=\frac{\beta(1-\beta)\left[\gamma_{\mu \varepsilon}^{2} \varepsilon_{c}(\lambda-1)+3 \lambda(\eta-1)\right]}{\varepsilon_{c}\left(\gamma_{\mu \varepsilon}^{2} \varepsilon_{c} \tilde{\lambda}-3 \lambda \tilde{\eta}\right)(6 \tilde{\eta}+4 q)} \\
+\frac{3}{2} \frac{\beta(1-\beta) \gamma_{\mu \varepsilon}(\lambda \eta-1)}{\left(\gamma_{\mu \varepsilon}^{2} \varepsilon_{c} \tilde{\lambda}-3 \lambda \tilde{\eta}\right)(6 \tilde{\eta}+4 q)},
\end{gathered}
$$

$$
\begin{gathered}
c_{l}=\frac{6\left[\beta+\eta^{2}(1-\beta)\right]+4 q \tilde{\eta}}{6 \eta(6 \tilde{\eta}+4 q)} \\
c_{s}=\frac{\tilde{\eta}\left(\gamma_{\mu \varepsilon}+\frac{3}{2}\right)(6 \eta+4 q)\left[\gamma_{\mu \varepsilon}^{2} \varepsilon_{c}(\beta-1)+3 \lambda \eta\right]}{6 \gamma_{\mu \varepsilon} \varepsilon_{c} \eta\left(\gamma_{\mu \varepsilon}^{2} \varepsilon_{c} \tilde{\lambda}-3 \lambda \tilde{\eta}\right)(6 \tilde{\eta}+4 q)} \\
-\frac{3 \tilde{\eta} \lambda \beta(\eta-1)\left[9 \eta+\gamma_{\mu \varepsilon}(6+4 q+6 \eta)\right]}{6 \gamma_{\mu \varepsilon} \varepsilon_{c} \eta\left(\gamma_{\mu \varepsilon}^{2} \varepsilon_{c} \tilde{\lambda}-3 \lambda \tilde{\eta}\right)(6 \tilde{\eta}+4 q)} \\
-\varepsilon_{c} \frac{\tilde{\eta} \lambda \beta \gamma_{\mu \varepsilon}(6+4 q)\left(\gamma_{\mu \varepsilon}+\frac{3}{2} \eta\right)}{6 \eta\left(\gamma_{\mu \varepsilon}^{2} \varepsilon_{c} \tilde{\lambda}-3 \lambda \tilde{\eta}\right)(6 \tilde{\eta}+4 q)} .
\end{gathered}
$$

We now express the fluxes in terms of their respective equilibrium temperatures as given by

$$
\mathcal{B}=\frac{\sigma_{\mathrm{SB}} T^{4}}{\pi}, \quad \mathcal{F}=\frac{\sigma_{\mathrm{SB}} T_{\mathrm{int}}^{4}}{\pi}, \quad \mathcal{F}_{*}=\bar{\mu} \frac{\sigma_{\mathrm{SB}} T_{\mathrm{irr}}^{4}}{\pi},
$$

where $\sigma_{\mathrm{SB}}$ is the Stefan-Boltzmann constant, $T_{\text {int }}$ is the internal temperature associated with the thermal flux at the bottom of the atmospheres and $T_{\text {irr }}$ is the irradiation temperature associated with the flux at the top of the atmosphere. This substitution results in the temperature profile given by

$$
\begin{aligned}
T^{4}= & \frac{3 T_{\mathrm{int}}^{4}}{4}\left[\frac{\eta}{\varepsilon_{c} \tilde{\eta}} \tau+\frac{4 \eta}{\varepsilon_{c} \tilde{\eta}} c_{l}-\frac{4(\eta \lambda-1)}{\tilde{\lambda}} b_{l} \exp (-q \tau)\right] \\
& -\frac{3|\bar{\mu}| T_{\mathrm{irr}}^{4}}{4}\left[\frac{4 \eta}{\varepsilon_{c} \tilde{\eta}} c_{s}-\frac{4(\eta \lambda-1)}{\tilde{\lambda}} b_{s} \exp (-q \tau)\right. \\
& \left.-\frac{\left(\gamma_{\mu \varepsilon}^{2} \varepsilon_{c}-3\right)\left(\gamma_{\mu \varepsilon}^{2} \varepsilon_{c}-3 \eta \lambda\right)}{3 \gamma_{\mu \varepsilon} \varepsilon_{s}\left(\gamma_{\mu \varepsilon}^{2} \varepsilon_{c} \tilde{\lambda}-3 \lambda \tilde{\eta}\right)} \exp \left(-\gamma_{\mu \varepsilon} \tau\right)\right]
\end{aligned}
$$

In the limit of $\beta \rightarrow 0$ and no external irradiation $T_{\text {irr }}=0$, we have

$$
\tilde{\eta}=\eta, \quad \tilde{\lambda}=1, \quad b_{l}=0, \quad c_{l}=\frac{1}{6},
$$

and Equation (51) reduces to the classic Milne's solution

$$
T^{4}=\frac{3}{4} T_{\text {int }}^{4}\left(\tau+\frac{2}{3}\right) .
$$

With only $T_{\text {irr }}=0$, we recover the nonirradiated solution of Chandrasekhar (1935, see Equation (52))

\section{Results}

The picket fence model of Chandrasekhar (1935) was originally developed to capture the effects that arise due to line blanketing in stellar atmosphere models. The initial treatment was based on the ideal case of lines with uniform width, strength, and separation. A discussion of the specific limitations that result from these assumptions is presented in Athay \& Skumanich (1969) and Athay (2012). Nevertheless, the original model did succeed in illustrating the basic effects due to line blanketing, namely that of cooler surface temperatures and warmer deeper temperatures. We have, therefore, retained the same simplified treatment of the lines in order to construct a general nongray model that includes coherent isotropic scattering. All of the results presented here are derived assuming fiducial values for the 

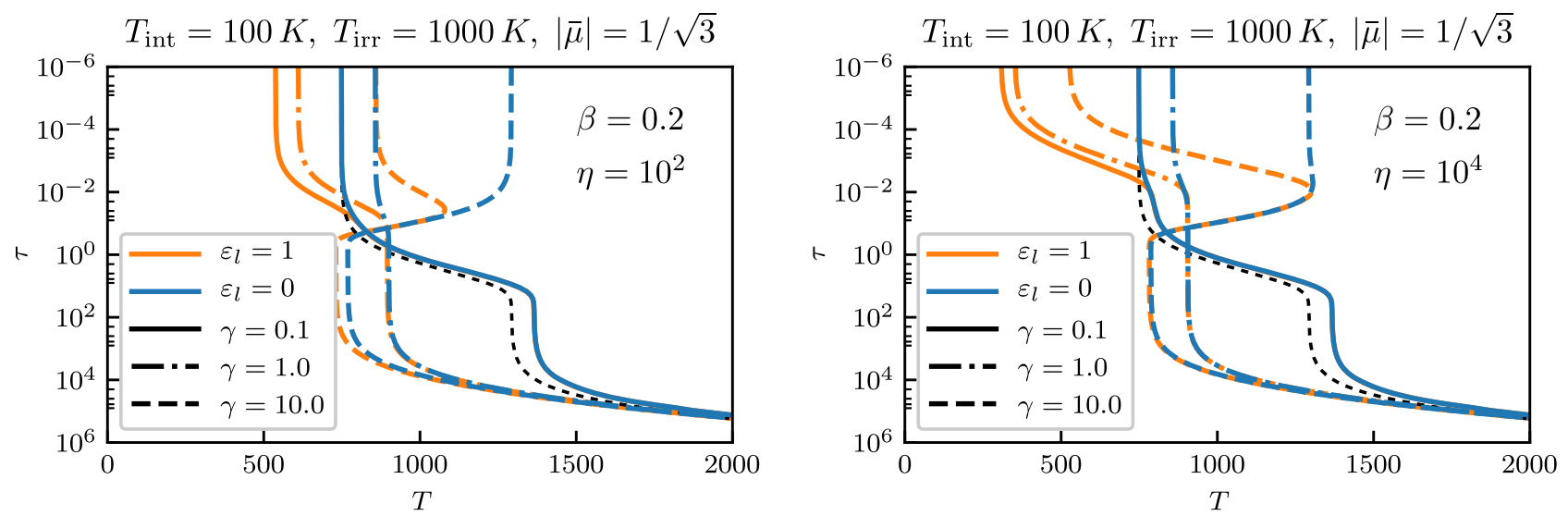

Figure 2. Temperature profiles for a given line width $\beta$ and two different values of the line strength $\eta$. The temperature profile in each case is derived for three different values of the shortwave opacity parameter $\gamma$ represented by the solid, dashed, and dashed-dotted curves. The blue curves represent the profile with lines that are due to pure scattering, whereas the orange curves represent lines due to pure absorption. $\gamma>1$ results in an anti-greenhouse effect leading to a relatively hotter upper atmosphere and a cooler lower atmosphere. The dotted black line is the gray temperature profile computed for $\gamma=0.1$ and is plotted here to illustrate the backwarming effect seen in the solid (blue and orange) curves for the same $\gamma$.

internal temperature $T_{\text {int }}=100 \mathrm{~K}$, the irradiation temperature $T_{\text {irr }}=1000 \mathrm{~K}$ and irradiation angle given by $|\bar{\mu}|=1 / \sqrt{3}$.

\subsection{Surface Cooling and Backwarming}

Line blanketing introduces two main effects in the thermal structure and spectrum of atmospheres ${ }^{4}$ If line formation can be attributed solely to absorption processes, the temperature of the upper layer is lower compared to what it would be in the absence of lines (the gray limit), an effect that is referred to as surface cooling (Athay 2012). The degree of surface cooling is lowered if the lines are partly due to scattering processes parameterized here by $\varepsilon_{l}$. The surface cooling is completely absent if the lines are entirely due to scattering $\varepsilon_{l} \rightarrow 0$. Figure 1 shows the change in boundary or skin temperature, here referred to as the temperature at zero optical depth, with the longwave scattering parameter $\varepsilon_{l}$ for a fixed line width but different line strengths. In the case of irradiated atmospheres, the strength of the shortwave opacity also influences the boundary temperature due to greater or lower relative absorption of incident starlight in the upper layers. Higher values of $\gamma$, therefore, lead to higher upper layer temperatures.

The deeper layer warming observed in the nongray model that we have derived here is due to the effect of line blanketing. This backwarming results from the increase in temperature due to an attendant increase in the radiative flux per unit interval that is redistributed within the continuum band of frequencies as a result of its occlusion by the lines. Deeper layer backwarming is, however, insensitive to the nature of line formation. Figure 2 shows temperature profiles for two limiting values of the scattering parameter $\varepsilon_{l}$ and two different values of the line strength $\eta$. The profiles are calculated for a fixed angle of the irradiation beam, a constant line width, fiducial values of the effective internal and irradiation temperatures, and three different values of the shortwave absorption opacity (excluding the effect of shortwave scattering here). We plot the gray temperature profile with $\gamma=0.1$ as a dotted black line in Figure 2 to illustrate the backwarming effect. Notice that the corresponding nongray temperature profile with $\varepsilon_{l}=1$ and $\varepsilon_{l}=0$ in Figure 2 are both warmer by the same extent with

\footnotetext{
4 Some works include the additional effect of line blocking, which we do not consider here. Our usage of the term blanketing is in the sense of Athay (2012).
}

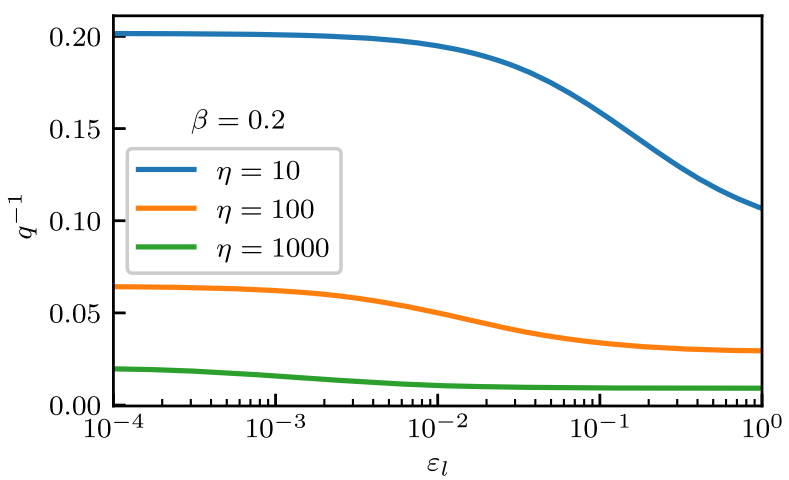

Figure 3. Characteristic optical depth $q^{-1}$, below which surface cooling effects are observed, as a function of the line scattering parameter $\varepsilon_{1}$ for different values of the line strength $\eta$ at a fixed line width $\beta$.

reference to the dotted line thereby illustrating the the deeper layer warming as well as its insensitivity to the nature of line formation. The degree of backwarming increases with the width of the line, represented here by $\beta$, and has been examined in detail by Parmentier \& Guillot (2014). In the limit $\varepsilon_{l}=1$ and $\gamma \gtrsim 1$, we see, as was also found in Parmentier \& Guillot (2014), that the lower boundary temperatures confine the heating due to stronger shortwave absorption into a thin hot layer immediately below the surface. This thin hot layer is of course absent in the opposite limit of $\varepsilon_{l}=0$ due to the lack of surface cooling.

\subsection{Limit Optical Depth}

A characteristic depth that emerges from the picket fence calculation is given in terms of $q^{-1}$ as defined by Equation (41). The corresponding quantity in Parmentier \& Guillot (2014) is referred to as $\tau_{\lim }$ and it represents the depth above which the surface cooling effect may be present in the atmosphere provided that the lines are due in some part to absorption processes. This scale is a function of the line width $\beta$, the line opacity ratio $\xi$, as well as the scattering parameters $\varepsilon_{l}$ and $\varepsilon_{c}$. However, as shown in Figure 3, the variation of $q^{-1}$ with respect to $\varepsilon_{l}$ is negligible and is largely decided by the line width and strength. Similarly, any meaningful change in $\varepsilon_{c}$ brings about only a negligible modification to $q^{-1}$. Our results derived for $\varepsilon_{l} \rightarrow 1$ are 

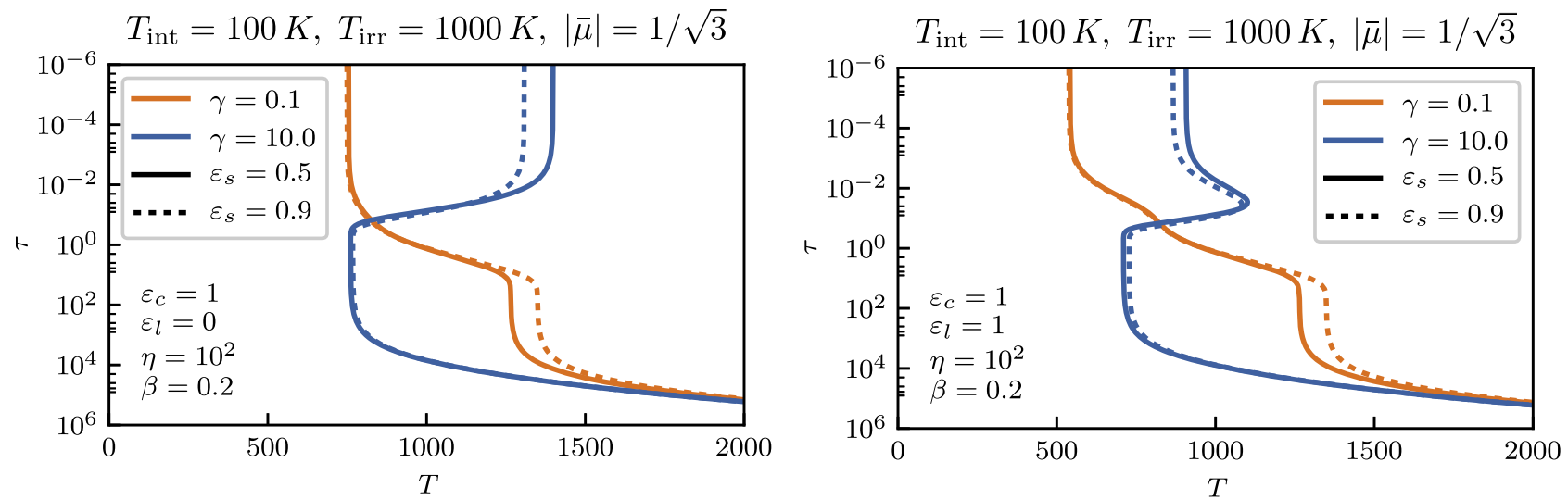

Figure 4. Temperature profiles for two different values of the shortwave continuum coherent isotropic scattering parameter $\varepsilon_{s}$, represented by the solid and dotted lines. The profiles are derived in the presence of spectral lines of a given width and strength as well as two different values of the shortwave opacity parameter $\gamma$ represented by the orange and blue curves. The left panel illustrates the temperature profile when the lines are due to pure scattering and the right panel is for lines due to pure absorption. We consider the limit of pure absorption in the longwave continuum here. The inclusion of coherent shortwave continuum scattering results in a leftward shift in the temperature profile toward cooler temperatures.
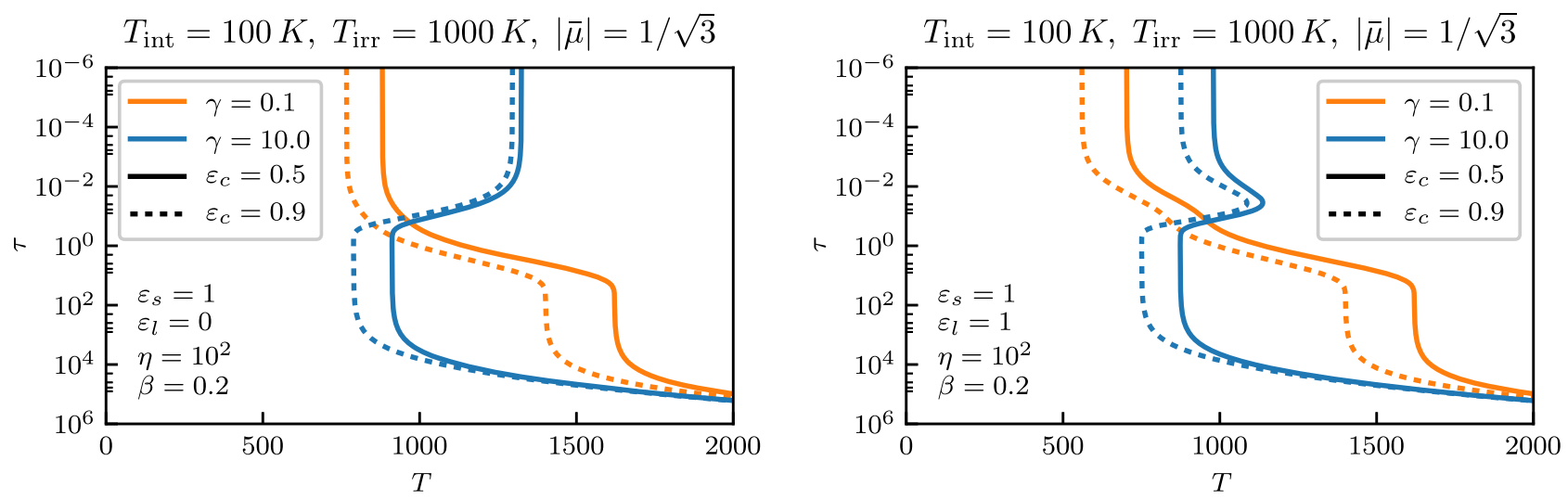

Figure 5. Temperature profiles for two different values of the longwave continuum coherent isotropic scattering parameter $\varepsilon_{c}$, represented by the solid and dotted lines. The profiles are derived in the presence of spectral lines of a given width and strength as well as two different values of the shortwave opacity parameter $\gamma$ represented by the orange and blue curves. The left panel illustrates the temperature profile when the lines are due to pure scattering and the right panel displays the profiles when the lines are due to pure absorption. We consider the limit of pure absorption in the shortwave continuum here. The inclusion of coherent longwave continuum scattering results in a rightward shift in the temperature profile toward hotter temperatures.

qualitatively similar to Parmentier \& Guillot (2014) and differ quantitatively only by a factor of a Rosseland mean opacity $\varrho$, defined here in dimensionless form as (Chandrasekhar 1935, see Equation (56))

$$
\frac{1}{\varrho} \equiv \frac{\beta}{\eta}+1-\beta .
$$

\subsection{Longwave and Shortwave Continuum Scattering}

The effect of coherent scattering in the shortwave is to push the temperature profile to lower values on a near global scale (Heng 2017). Coherent isotropic continuum scattering in the shortwave is parameterized in terms of $\varepsilon_{s}$ and its effect on the temperature profile is demonstrated in Figure 4. The global shift in the thermal profile toward lower temperatures adds to any surface cooling present that is due to absorption lines and also effectively offsets the backwarming in the mid to deeper layers. The lower temperatures result from a reduction in the total energy budget by a factor of $1-A_{\mathrm{B}}$, where $A_{\mathrm{B}}$ is the Bond albedo, which may be expressed in terms of the scattering parameter $\varepsilon_{s}$ (Heng et al. 2012).

Coherent isotropic scattering in the longwave continuum band of frequencies has a similar atmosphere-wide effect where the temperatures are now shifted to higher values as illustrated in Figure 5. This is a manifestation of the classical greenhouse effect (Heng 2017) and is different from the lack of surface cooling due to lines formed by scattering. The former is an actual warming process and is present on a global scale, whereas the latter is the result of the lines being uncoupled from the thermal energy reservoir (Mihalas \& Luebke 1971). Taken together, scattering processes therefore play an important role in determining the equilibrium temperature profile even in simple pseudo-nongray models.

\section{Summary}

We have derived an analytical model for irradiated atmospheres that combines the effect of spectral lines in the longwave band of frequencies, where the lines may be due to either pure absorption or pure coherent scattering processes or some combination of the two. To achieve this, we adapted the picket fence treatment of Chandrasekhar (1935) to model line blanketing effects. The picket fence treatment has been recently used to model irradiated atmospheres but without including the possibility of lines due to coherent scattering (Parmentier \& Guillot 2014). Our results demonstrate that the cooling of the upper layers due to line blanketing depends on the nature of 
line formation as was previously observed in the context of nonirradiated atmospheres. If scattering is solely responsible for the lines, then the surface temperatures retain their gray value, as the lines are not coupled to the thermal energy of the gas in this limit. Transit spectroscopy of exoplanets is generally most sensitive to very low pressure levels or equivalently the upper layers of the exoplanet's atmosphere (Madhusudhan et al. 2014). Given that the surface temperature is sensitive to the line formation process as revealed by the picket fence analysis, one must exercise caution in the interpretation of observations on the basis of atmospheric transfer models. Furthermore, the contribution of coherent scattering in the continuum can significantly alter global temperature levels depending on the wavelength band. If the planet reflects some fraction of its incident light, the deeper layer temperatures are lowered and negates the backwarming effect due to the lines. If coherent scattering is present in the longwave continuum, the greenhouse effect comes into play leading to greater warming throughout the atmosphere. Our analytical model therefore accommodates a greater range of possibilities over a larger parameter space and may be used to derive reasonable estimates of the thermal structure of irradiated atmospheres.

G.M. thanks the Center for Space and Habitability in Bern, where part of this work was carried out, for their hospitality. G.M. thanks the Kavli Institute for Theoretical Physics, where this work was completed, for their hospitality and support through the KITP graduate fellowship program. The research leading to these results has received funding from the European Research Council under the European Unions Seventh Framework Programme (FP/2007-2013) under ERC grant agreement 306614. K.H. thanks the Swiss National Science Foundation, the Center for Space and Habitability, the European Research Council, and the Swiss-based MERAC Foundation for partial financial support. This research was supported in part by the National Science Foundation under Grant No. NSF PHY $17-48958$.

\section{ORCID iDs}

Gopakumar Mohandas (ㄴ) https://orcid.org/0000-00021212-3492

Martin E. Pessah (1) https://orcid.org/0000-0001-8716-3563

Kevin Heng (i) https://orcid.org/0000-0003-1907-5910

\section{References}

Athay, R. G., \& Skumanich, A. 1969, ApJ, 155, 273

Athay, R. G. 2012, Radiation Transport in Spectral Lines, Vol. 1 (Berlin: Springer Science \& Business Media)

Chandrasekhar, S. 1935, MNRAS, 96, 21

Guillot, T. 2010, A\&A, 520, A27

Hansen, B. M. S. 2008, ApJS, 179, 484

Heng, K. 2017, Exoplanetary Atmospheres: Theoretical Concepts and Foundations (Princeton, NJ: Princeton Univ. Press)

Heng, K., Hayek, W., Pont, F., \& Sing, D. K. 2012, MNRAS, 420, 20

Heng, K., Mendonça, J. M., \& Lee, J.-M. 2014, ApJS, 215, 4

Hubeny, I. 2017, MNRAS, 469, 841

Hubeny, I., \& Mihalas, D. 2014, Theory of Stellar Atmospheres (Princeton, NJ: Princeton Univ. Press)

Madhusudhan, N., Knutson, H., Fortney, J. J., \& Barman, T. 2014, in Protostars and Planets VI, ed. H. Beuther et al. (Tucson, AZ: Univ. of Arizona Press), 739

Mihalas, D. 1970, Stellar Atmospheres (San Francisco, CA: Freeman)

Mihalas, D., \& Luebke, W. R. 1971, MNRAS, 153, 229

Münch, G. 1946, ApJ, 104, 87

Parmentier, V., \& Guillot, T. 2014, A\&A, 562, A133 